\title{
Functional Profiling and Future Research Direction of Rice Bran Oil in Bangladesh
}

\author{
Afroza Sultana1, Mohammad Ali Zinnah², Habibul Bai Shozib³ ${ }^{3}$ Zakir Hossain Howlader², \\ and Md Alauddin ${ }^{1 *}$

\footnotetext{
${ }^{1}$ Department of Nutrition and Food Technology, Jashore University of Science and Technology, Jashore-7408, BANGLADESH

${ }^{2}$ Department of Biochemistry and Molecular Biology, University of Dhaka, Dhaka-1000, BANGLADESH

${ }^{3}$ Bangladesh Rice Research Institute (BRRI), BANGLADESH
}

\begin{abstract}
Rice bran oil (RBO) has been demonstrated to affect complex malfunctioned conditions such as oxidative stress, hyperlipidemia, hyperglycemia, hypertension, inflammation, abnormal cell growth (cancer), ulceration, immune and cognitive modulation. This unique effect of RBO is due to the presence of well-balanced fatty acid composition and several bioactive compounds, $\gamma$ - oryzanol (cycloartenyl ferulate, 24-methylenecycloartanyl ferulate, campesterol ferulate, and $\beta$-sitosteryl ferulate), vitamin $E$ (tocopherol and tocotrienol), phytosterols ( $\beta$-sitosterol, campesterol and stigmasterol) and other nutrients. The RBO composition of bioactive compounds varied geographically, thus the clear-cut mechanisms of action on complex disease cascades are still required. This review article summarized the RBO compositional profiling and compared it with other edible oils. This article also summarized Bangladesh RBO profiling and their proposed mechanism of action as well as the first line of defense in the prevention, management, and control of complex disease conditions. This review indicates how Bangladesh RBO increase their opportunity to be functional food for 21 st century's ailment.
\end{abstract}

Key words: rice bran oil (RBO), composition, Bangladesh RBO, antioxidant, anti-inflammatory, anticancer and anti-ulcerogenic

\section{Introduction}

A short flashback of the history of rice grown in the world, domesticated rice species were grown worldwide among them Oryza sativa and Oryza glaberrima is called Asian rice and African rice respectively. Asian cultivars extensively cultivated Oryza sativa Japonica and Oryza sativa Indica is the history of about 13000 years ago ${ }^{1)}$. The species Oryza sativa Indica comes from the crosses between Oryza sativa Japonica and local wild rice. The genetic evolution, geographical diversity, land quality, different agronomic practices, and environmental conditions may cause nutritional variation among global rice. The composition of whole rice grain has been divided into several compartments such as edible part (70\%), non-edible hull (20\%), bran $(7 \%-8.5 \%)$, and rudiment $(2 \%-3 \%)^{2)}$. Rice bran oil(RBO), extracted from rice bran, is gained popularity as a healthy cooking oil in Japan, India, Indonesia, Korea, Thailand, China, Vietnam, and Bangladesh. The
RBO is obtained from rice bran through different extraction processes, among them, solvent extraction(using hexane) and mechanical pressing (cold pressing) is the most popular and conventional method for commercial extraction $^{3)}$. The percentage of yield and nutritional value of RBO may vary according to the chemical composition of bran, rice variety, treatment of the grain before milling, the technology of milling, degree of milling, and the downstream

Abbreviations: RBO; Rice bran oil, $\boldsymbol{\gamma}$ - oryzanol; Gamma oryzanol, CLA; Conjugated linoleic acid, SFA; saturated fatty acid, MUFA; monounsaturated fatty acid, PUFA; polyunsaturated fatty acid, $\boldsymbol{\alpha}$; alpha, $\boldsymbol{\beta}$; beta, $\boldsymbol{\gamma}$; gamma, $\boldsymbol{\delta}$; delta, FFA; free fatty acids, P-AMPK; phosphorylation of AMPactivated protein kinase, PPAR- $\boldsymbol{\gamma}$; peroxisome proliferatoractivated receptor-gamma, NF-kb; nuclear transcription factorkappa B, TNF- $\alpha$; tumor necrosis factor-alpha, iNOS; inducible nitric oxide synthase NO; nitric oxide, COX-2; cyclooxygenase-2, mPGES-1; microsomal prostaglandin E synthase-1

\footnotetext{
*Correspondence to: Md. Alauddin, Department of Nutrition and Food Technology Jashore University of Science and Technology, Jashore-7408. BANGLADESH

E-mail: mdalauddin_nft@just.edu.bd, mdalauddin1181@gmail.com ORCID ID: https://orcid.org/00000001-8850-8025

Accepted September 21, 2021 (received for review June 28, 2021)

Journal of Oleo Science ISSN 1345-8957 print / ISSN 1347-3352 online

http://www.jstage.jst.go.jp/browse/jos/ http://mc.manusriptcentral.com/jjocs

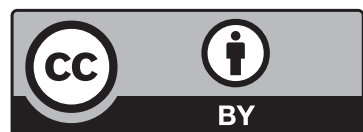


processing of $\operatorname{bran}^{4,5)}$. This review article thoroughly discussed the functional profiling of $\mathrm{RBO}$ and the future research direction of RBO in Bangladesh.

\section{Components and Bioactivities of Rice Bran Oil (RBO)}

The oil content of rice bran depends on various factors. For instance, glutinous rice contains more oil than non-glutinous rice, and parboiled rice contains higher oil content than non-parboiled rice because the oil content (lipid droplets) shifted to the bran layer from the aleurone layer during parboiling. The oil content of rice bran varies between $10 \%$ and $23 \%$ depending on extraction methods $^{4)}$. Different organizations, associations, institutes, and society have endorsed that RBO is a healthy oil because of its balanced fatty acid composition in the saponifiable compartment (0.6:1.1:1) saturated fatty acid (SFA) : monounsaturated fatty acid (MUFA) : polyunsaturated fatty acid (PUFA) respectively, and other bioactive components present in the unsaponifiable fraction of $\mathrm{RBO}^{6,7}$. $\mathrm{RBO}$ is a rich source of bioactive compounds, especially $\gamma$-oryzanol (which is rarely found in other edible oils. However, the content of bioactive compounds depends on the refining process of RBO. In previous studies, it has been observed that RBO contains 1.8\%-2.2\% $\gamma$-oryzanol, $0.04 \% \alpha$-tocopherol, and a lesser amount $\gamma$-tocopherol, $0.07 \% \gamma$-tocotrienol. RBO also contains squalene and phytosterols, including $\beta$-sitosterol, campesterol, stigmasterol, and isofucosterol ${ }^{8}$. The fatty acid composition of RBO surprisingly is enabled to reduce body total cholesterol(TC), low-density lipoprotein cholesterol(LDL-C), and apolipoprotein $\mathrm{B}(\mathrm{ApoB})$, which was observed in a previous study $^{9)}$.

The antilipidemic effect of RBO has been observed by its PUFA because the PUFA is claimed to have anti obese, antidiabetic, and antihypertensive properties ${ }^{10,11)}$. The bioactive compound $\gamma$-oryzanol have a similar antilipidemic effect but there is no significant difference between a low and high dose of $\gamma$-oryzanol for atherosclerotic condition ${ }^{12)}$. $\mathrm{RBO}$ can reduce atherosclerotic conditions because it has not only $\gamma$-oryzanol but also other micronutrients like tocopherol, tocotrienol, and phytosterols like campesterol, stigmasterol, and beta-sitosterol have the synergistic effect with $\gamma$-oryzanol ${ }^{12,13)}$. The macro components (various lipid compounds) and micronutrients ( $\gamma$-oryzanol, vitamin E, phytosterols, squalene, policosanol) of RBO are effective against hyperlipidemia, hypertension, hyperglycemia, cancer, oxidation, inflammation, insomnia, and immunomodulation that are summarized in Fig. 1.

The fatty acid content of RBO greatly varies with their rice bran genotype and environmental factors such as temperature. Generally, RBO contains 19\%-35\% SFA including palmitic acid, myristic acid, stearic acid, arachidic acid, etc., and 55\%-87\% unsaturated fatty acids(UFA) such as oleic acid, linoleic acid, and linolenic acid, which are greatly affected by the variety and agronomic conditions. For example, glutinous rice contains more oil as well as more SFA than non-glutinous. The fat composition is also affected by downstream processing technology. For instance, a large portion of SFA than unsaturated ones are removed during the $\mathrm{RBO}$ dewaxing process. The triacylglycerides (TAG) of RBO vary between $3.4 \%$ and $49.9 \%$ are triunsaturated. The free fatty acids (FFA) come from TAG by the action of lipase, which can cause a bad flavor of RBO. The physical and chemical(ohmic heat and ethanol) treatment of rice bran before $\mathrm{RBO}$ processing limited the

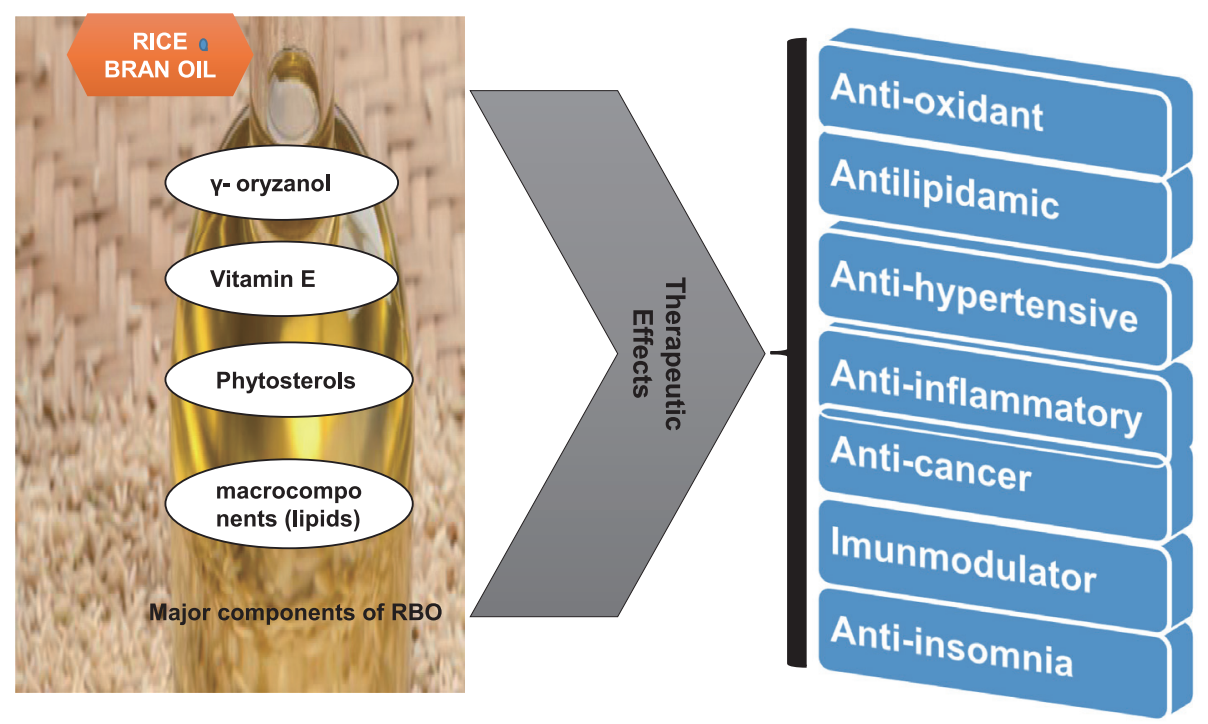

Fig. 1 The major bioactive components of rice bran oil(RBO) and their therapeutic effects. 
Table1 Macromolecule and micromolecule composition of RBO(adopted from reference 2,17-20).

\begin{tabular}{lc}
\hline \multicolumn{1}{c}{ Name of components } & Percentage (\%) \\
\hline Gama oryzanol & $1.8-2.2$ \\
Vitamin A & $0.001-0.008$ \\
Tocopherol & 0.04 \\
Tocotrinol & 0.07 \\
Phytosterols & $0.8-1.3$ \\
Triacylglycerol & $81-84$ \\
Diacylglycerol & $2-3$ \\
Monoacylglycerol & $1-2$ \\
Free fatty acid & $2-6$ \\
Wax & $3-4$ \\
Phospholipid & $1-2$ \\
Saturated fatty acid & $18.4-25.5$ \\
Mono unsaturated fatty acid & $38.4-42.3$ \\
Poly saturated fatty acid & $33.6-39.2$ \\
\hline
\end{tabular}

FFA between $2 \%$ and $5 \%$ without hampering the other bioactive compounds ${ }^{14,15}$. Ten components of $\gamma$-oryzanol have been isolated and identified, among them four principal components, cycloartenyl ferulate (11.40\% ), 24-methylenecycloartanyl ferulate (50.20\%), campesteryl ferulate (24\%), and beta-sitosteryl ferulate(14.40) were found in the Bangladeshi rice bran variety ${ }^{16)}$. Other bioactive components (tocopherol and tocotrienol each have four major types of categories) comprise $0.1 \%-0.2 \%$ and phytosterols (primarily $\beta$-sitosterol, campesterol, stigmasterol) $1.5 \%$ $-2.0 \%$ respectively. The most desirable RBO accounts for a thin and clear appearance, high bioactive components, and lower level of $\mathrm{FFA}^{17)}$. The macro and micro molecule compositions are summarized in Table $1^{2,17-20)}$.

\section{Properties of Rice Bran Oils (RBO) with Other Edible Oils}

Comparison of RBO in contrast to some physical and chemical aspects of other edible oils that are summarized in Table 2. This comparison has pointed out several cases, making RBO in a remarkably better position than other edible oils. RBO is healthier than soybean oil, palm oil, mustard oil, and olive oil. It is important to pay attention to the smoke points of various fats, as described fats are no longer suitable for consumption after they exceed the smoke point and begin to break down. The oils, that are listed in Table 2, are preferably used for cooking, frying, baking, salads, and seasoning. RBO is a very clean and pleasant-tasting oil with attractive color and a mild taste.
Due to its very high smoke point $\left(\sim 234^{\circ} \mathrm{C}\right)$ and flashpoint $\left(\sim 350^{\circ} \mathrm{C}\right)$, it is very stable and has almost zero degradation and polymerization during cooking. The high smoke and special bioactive compound of RBO make it an ideal oil for all types of cooking, ranging from stewing to deep-frying. Considering this point, rice bran oil may be a better substitute. The attractive color of RBO ranging from yellow-green to dark brown depends on certain pigmented substances such as carotenoid, lutein, and gum. The refining process of $\mathrm{RBO}$ removes the unexpected substance and can produce the color of $\mathrm{RBO}$ lighter, even though the refining process cannot completely remove the pigmented substance like other vegetable oils. On the other hand, freshly prepared soybean oil is lighter. However, the color of the oil sometimes darkens during storage due to its oxidation. Olive oil naturally has a variety of colors, ranging from light yellow to dark gold due to its pigmented substance content. Lower peroxide value (PV) describes the freshness of the lipid matrix during the storage of soybean oil and RBO. A high peroxide value(PV) is an indicator of an oxidation state. The more oxidized oil produces higher $\mathrm{PV}$, which indicates the lower freshness of the oil matrix during storage ${ }^{21)}$. Density is an important factor that influences oil absorption as it affects the drainage rate after frying and also the mass transfer rate during the cooling stage of frying ${ }^{22)}$. The oil density ranging from 0.91 to 0.93 $(\mathrm{g} / \mathrm{L})$ depends on the nature of the oil at a temperature of $15^{\circ} \mathrm{C}$ to $25^{\circ} \mathrm{C}$ comparing it with a water density of $1.00 \mathrm{~g} /$ $\mathrm{mL}$. The RBO is said to be better for cooking due to its density smaller than other vegetable oils. The iodine value describes the unsaturation of oil, and the average amount of double bonds present in the oil. A decrease in the number of double bonds indicates oil is oxidized during storage time. Oxidative stability is another parameter for oil quality. A study of vegetable oil after frying food showed that soybean oil significantly reduced the iodine value after frying and storage of the oil. This indicates that the fat oxidation was relatively high for soybean oil compared to other vegetable oils such as palm oil and peanut oil. The frying and storage conditions have a significant impact on oil quality ${ }^{23)}$. The RBO has a higher induction time due to its antioxidant properties. The phenolic compounds are very important to protect PUFA present in RBO. Thus, the high quantity of phenolic compounds (ferulic acid, erucic acid, cinnamic acid, dimethyl sterol, monomethyl sterol) is responsible for the oxidation stability of RBO. Another cause of the oxidation stability of RBO is the quantity of vitamin $\mathrm{E}$ which exists as a mixture of four distinct types of monophenols (such as tocols) contain four homologues (Alpha, Beta, Gamma, Delta) of tocopherols and four diverse types of tocotrienols (Alpha, Beta, Gamma, Delta) having three double bonds on the side chain with -tocopherol serving as the methylene group. One of the studies provides an excellent overview of the percentages of differ- 


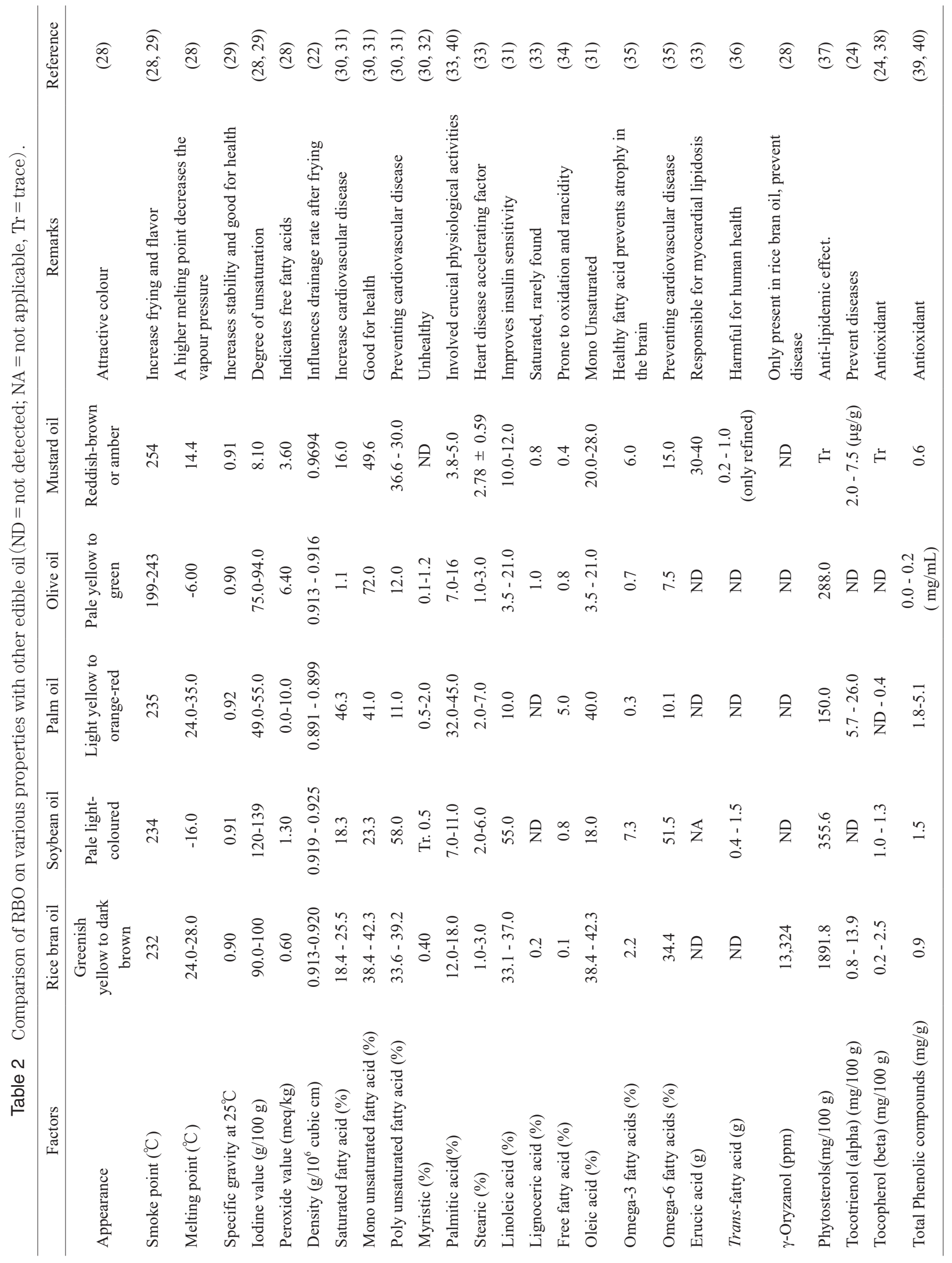


ent tocos homologues found in the majority of popular vegetable oils, including $\mathrm{RBO}^{24}$. Additionally, $\mathrm{RBO}$ includes all of the tocotrienol categories and homologues, but the four tocotrienol forms are not detected/traced in the majority of other cooking oils, including soybean oil, olive oil, sunflower oil, sesame, and safflower (except palm oil). Even though it is commonly found in most cooking oils, but RBO contains a well amount of tocopherol and tocotrienol. Among their eight stereoisomers (alpha, beta, gamma, and delta), only two stereoisomers may be more active or more sensitive than others. They are potent antioxidants and function differently from each other due to the presence of three double bonds at the $3^{\prime}, 7^{\prime}$ and $11^{\prime}$ positions in their side chains ${ }^{24)}$. For instance, tocotrienol has neuroprotective, antioxidant, anti-cancer, and cholesterol-lowering properties than tocopherol because tocotrienol can penetrate more effectively into tissues with saturated fat layers in the brain and liver ${ }^{25}$. One of the important components of RBO is phytosterol, a compound that prevents dietary cholesterol absorption, thereby reducing the body's cholesterol. The unrefined RBO contains a higher amount of phytosterol (848-1034 mg / $100 \mathrm{~g}$ that can lower LDL cholesterol and triacylglycerol levels but increase HDL. The RBO is a good source of linoleic and oleic fatty acids, fat-soluble vitamins, and phytosterols rather than other vegetable oils $^{26)}$. RBO contains appreciable quantities of bioactive components and has attained the status of "Heart oil" due to its cardiac-friendly chemical profile ${ }^{27)}$. Finally, the trump card bioactive component of RBO is $\gamma$-oryzanol, a mixture of esters of ferulic acid, sterol, and triterpene. It was claimed that RBO is "The Healthiest Oil," nevertheless the nutritional profile is identical to other oils, there are just a few differences over other oils. One might be a high source of MUFA (44\%), PUFA (33.6\%), tocopherol, tocotrienol, and phytosterol, making it a popular cooking oil in Asia and tropical nations. The physiochemical parameters of RBO are almost similar to the widely used edible oil including soybean, palm, olive, mustard oil etc. High smoke point is an important parameter for any edible oil as it starts to break down, altering its flavor and releasing free radicals at that point. After the smoke point, a substance called acrolein makes the oil taste burnt and bitter. RBO is suitable for high-temperature frying. $\mathrm{RBO}$ is a unique edible oil with numerous health benefits. The presence of a very small amount of Omega-3 fatty acids ( $\alpha$-Linolenic acid) compared to other edible oil is the main limitation of this oil ${ }^{6,28-39)}$. However, RBO is a rich source of omega-6 (linoleic acid), which is an essential fatty acid similar to omega-3, because these are not synthesized by the human body and come from food sources. Several Countries have recommended allowances of fat consumption and set the absolute values of PUFAs including balanced intake of Omega- 6 and Omega-3. RBO meets the ratio of polyunsaturated fatty acid and saturated fatty acids, linoleic acid, and $\alpha$-linolenic acid in the diet 0.8 to 1.0 and 5-10 respectively (WHO/FAO, 2003). RBO is also a source of bioactive compounds such as flavonoid, sterol, and tocopherol are shown in Table 2. These substances are antioxidants, therefore inhibit the formation of free radicals, preventing chronic diseases. The presence of a huge amount of free phytosterols including gamma oryzanol, $\beta$-Sitosterol, campesterol, stigmasterol, isofucosterol in RBO makes this oil unique for preventing cholesterol levels. Thus, the comparison of RBO with other edible oil has pointed out a remarkably advantageous position than other edible oil.

\section{Rice Bran Oil Profiling and Future Research Directions in Bangladesh}

Flashback of rice bran oil history in Bangladesh, latter half of the mid-1970 century. Nobody could ever think that rice could be a source of oil for everyday consumption or would be worthwhile to use for practical purposes. At that time, people were accustomed to some of the oil crops such as mustard, sesame, and linseed grown locally, but this scenario was replaced by the cheap soybean and palm oil imported from western countries and Malaysia, respectively. The first RBO manufacturing company was established in the 1980s. Unfortunately, their production of RBO came to a halt for unknown reasons. Since then, 35 years have passed. Now 20 companies have licensed for RBO production in Bangladesh. Only 7 are in continuous production and 3 companies have completely stopped RBO production. Bangladesh produces 3.6 million tons (MT) of rice bran annually. Average RBO production in Bangladesh accounts for 250-300 tones daily based on $6.5 \mathrm{~kg}$ of rice bran being used to produce 1.0 liter of $\mathrm{RBO}^{40}$. The requirement for edible oil consumption is about 15 MT for the 160 million population in Bangladesh, according to $9.7 \mathrm{~g} / \mathrm{capita} /$ annum. Our existing resources of edible oil such as mastered seeds, sesame seeds, and groundnuts provide about 2.1 MT. Therefore, the remaining requirement of $13 \mathrm{MT}$ comes from abroad, western countries, and Malaysia. Soybean and palm oil is imported by drainage of our hardly-earn foreign currency to meet our demand. Currently, $\mathrm{RBO}$ is produced by our existing auto rice mill about 0.15 $\mathrm{MT}$, if we use our potential facilities for RBO production we can produce about 0.75 MT. Unless initiatives are taken to increase the domestic production of edible rice bran oil from domestic rice bran, there will be more drainage of foreign currency to meet the increased demand in our sustainable development goal $2030^{41-43)}$. Rice bran oil from different varieties in Bangladesh(BR-11, BRRI dhan-28, BRRI dhan-29, BRRI-48, and BRRI dhan-49 have been examined and were found that saturated fatty acid 16-21\% (mostly palmitic acid 14-18\%), monounsaturated fatty acid 39-49\% (mostly oleic acid 49\%) and polyunsaturated 
fatty acid 33-38\% (mostly linoleic acid 32-37\%). Constitutional variations were observed in the variety of sources of RBO. Differentiation of fatty acids composition in RBO was obtained from the bran of nine distinct types of rice commonly cultivated in Bangladesh along with four countries including Brazil, Thailand, Vietnam, and Japan. Fatty acid profiling of popular high-yielding rice varieties presented in Table 3 indicated that the range of saturated fatty acids 16.69 to $28.30 \%$ and unsaturated fatty acids 71.06 to 83.41\% whereas, monounsaturated fatty acids from 39.82 to $49.95 \%$ and polyunsaturated fatty acids from 33.24 to $38.58 \%$. Common fatty acids including linoleic acid from $28-35 \%$, linolenic acid variation was observed from 0.80 $2.69 \%$, and stearic acid from $1.59-4.21 \%$ indicated the variation is not drastic in case of variation of sources ${ }^{44-46)}$. The bioactive compound $\gamma$-oryzanol found $1.2-1.8 \%$, specifically 24 -methylene cycloartenol ferulate $50.20 \%$, cycloartenyl ferulate $11.40 \%$, campesteryl ferulate $24 \%$ and $\beta$-sitosteryl ferulate $14.40 \%$ among the $\mathrm{RBO}$ varieties in Bangladesh. Bioactive component Vitamin-A was also analyzed in different rice bran oil varieties and it was found 1.2-7.5 ppm, which was lower than the standard value of $(15-30 \mathrm{ppm})^{16,18)}$. The fatty acid profile of RBO and other edible oils that are marketed in Bangladesh have been analyzed. The result showed that saturated fatty acid was $16.62 \%$ in RBO, whereas it was $12 \%$ in soybean oil and $22 \%$ in butter oil. The monounsaturated fatty acid is 48.32 $\%$ in RBO, whereas it is $37 \%$ in soybean oil and $35 \%$ in butter oil. The polyunsaturated fatty acid is $35.06 \%$ in $\mathrm{RBO}$, whereas it is $28 \%$ in mustard oil and $25 \%$ in butter oil. Among them, balanced fatty acid composition and the highly bioactive compound $\gamma$-oryzanol found in Bangladesh rice bran oil would aid cardiovascular disease. It has been proved by an animal experiment on the long Evan rat model, this study showed that consecutive 28 days (about 4 weeks) supplementation of RBO significantly reduced body weight, plasma cholesterol, low-density lipoprotein choles- terol, and increased high-density lipoprotein cholesterol. There was no detrimental side effect on cardiac, renal, and kidney function. Moreover, the chronic supplementation of RBO on Swiss albino mice for 60 days significantly increased the erythrocytes and hemoglobin levels and reduced lipid profiles. Even though oils are detrimental to health, $\mathrm{RBO}$ is effective against lifestyle-related diseases ${ }^{46-48)}$. The RBO quality in Bangladesh is improving according to composition and health benefits, but the production facilities are limited. About 17 industries are available for RBO production in Bangladesh, but few are capable of refined RBO marketing. Thus, Bangladesh is trying to increase three times more RBO production capacity and its health benefits research to minimize the dependency on imported edible oil and save foreign currency. The researchers are paying attention to resolve the insight mechanism of (a) how Bangladeshi RBO itself or its bioactive compound reduces the lipid profile. (b) Whether the RBO can reduce the glucose level and how? (c) Whether and how does the RBO modulate cancer cells in vivo and in vitro? We have discussed our proposed mechanism of how RBO shows its properties in diabetics and hyperlipidemia in this review article.

\section{Properties of RBO in Diabetics and Hyperlipidemia}

The positive effect of RBO on diabetics and hyperlipidemia depends on the available bioactive components $\gamma$-oryzanol and tocotrienol. Diabetes, whether it is insulinindependent or dependent (both type-I and type-II), results in glucose impairment and creates undesirable oxidative stress at the cellular and molecular levels. Studies have demonstrated that the bioactive compounds of RBO reduce oxidative stress and diabetes, suggesting that the compounds themselves or their metabolites modulate cellular or molecular biomarkers related to diabetes ${ }^{48)}$. The

Table 3 Comparison of fatty acid composition(\%) of Bangladesh rice bran oil(RBO) with Reverence country's RBO (adopted form reference 45-47).

\begin{tabular}{|c|c|c|c|c|c|c|c|c|c|c|c|c|}
\hline & \multicolumn{8}{|c|}{ Common Rice Varieties in Bangladesh used for RBO } & \multicolumn{4}{|c|}{ RBO from Reverence country } \\
\hline & BR-5 & BR-10 & BR-11 & BRRI-28 & BRRI-29 & BRRI-39 & BRRI-48 & BRRI-49 & Brazil & Thiland & Vietnam & Japan \\
\hline Arachidic acid & $\sim 2.17$ & $\sim 2.11$ & $\sim 0.70$ & $\sim 0.54$ & $\sim 0.75$ & $\sim 1.01$ & $\sim 0.83$ & $\sim 0.97$ & ND & ND & ND & ND \\
\hline Behenic acid & ND & ND & $\sim 0.33$ & ND & ND & ND & $\sim 0.38$ & ND & ND & ND & ND & ND \\
\hline Eicosenoic acid & ND & ND & $\sim 0.44$ & ND & ND & ND & $\sim 0.46$ & $\sim 0.44$ & ND & ND & ND & ND \\
\hline Lauric acid & ND & ND & ND & $\sim 0.66$ & ND & ND & ND & ND & ND & ND & ND & ND \\
\hline Linoleic acid & $\sim 28.45$ & $\sim 30.84$ & $\sim 35.98$ & $\sim 32.13$ & $\sim 32.58$ & $\sim 31.15$ & $\sim 32.66$ & $\sim 37.26$ & $\sim 34.38$ & $\sim 33.41$ & $\sim 32.09$ & $\sim 35.04$ \\
\hline Linolenic acid & $\sim 0.80$ & $\sim 1.92$ & $\sim 1.21$ & $\sim 1.11$ & $\sim 0.88$ & $\sim 1.25$ & $\sim 1.22$ & $\sim 1.32$ & $\sim 2.69$ & $\sim 2.09$ & $\sim 2.18$ & $\sim 2.05$ \\
\hline Myristic acid & $\sim 0.45$ & $\sim 0.42$ & $\sim 0.24$ & $\sim 0.61$ & ND & $\sim 0.52$ & $\sim 0.44$ & $\sim 1.00$ & ND & ND & ND & ND \\
\hline Oleic acid & ND & ND & $\sim 43.16$ & $\sim 44.7$ & $\sim 49.95$ & ND & $\sim 45.36$ & $\sim 39.39$ & $\sim 40.13$ & $\sim 40.47$ & $\sim 41.99$ & $\sim 42.90$ \\
\hline Palmitic acid & $\sim 19.08$ & $\sim 19.86$ & $\sim 16.43$ & $\sim 18.39$ & $\sim 10.10$ & $\sim 20.86$ & $\sim 16.74$ & $\sim 17.44$ & $\sim 20.15$ & $\sim 21.04$ & $\sim 20.42$ & $\sim 17.22$ \\
\hline Stearic acid & $\sim 4.15$ & $\sim 4.01$ & $\sim 1.52$ & $\sim 1.78$ & $\sim 1.74$ & $\sim 3.40$ & $\sim 1.91$ & $\sim 2.18$ & $\sim 1.62$ & $\sim 1.93$ & $\sim 1.86$ & $\sim 1.57$ \\
\hline
\end{tabular}

ND. Not determined 
upstream signal of glucose metabolism is regulated by adiponectin, which is secreted by adipocytes. The level of adiponectin decreased in obesity and increased the lipogenesis and glucose levels in insulin-sensitive tissue, liver, and muscle. The adiponectin modulates the signal transduction of glucose utilization by translocation of glucose transporters (GLUT-2 and GLUT-4) to the cell surface and activation of the insulin receptor. A study showed that glucose level and lipogenesis are reduced by $\gamma$-oryzanol supplement because of its ability to increase adiponectin in obese patients. The bioactive component of RBO, $\gamma$-oryzanol induces the adipocyte to increase adiponectin secretion, which in turn increases the phosphorylation of AMP-activated protein kinase (P-AMPK) and inhibits gluconeogenesis and lipogenesis pathways in the liver ${ }^{49)}$. On the other hand, P-AMPK increased fatty acid oxidation by the activation of the transcription factor peroxisome proliferator-activated receptor-gamma $(\mathrm{PPAR}-\gamma)^{50,51)}$. RBO can reduce body weight by reducing body fat mass. The decreased fat mass can increase adiponectin secretion from adipocytes. The full length of adiponectin can bind to the adiponectin receptors (adipo-R1 and adipo-R2) and subsequently increases fatty acid oxidation and decreases gluconeogenesis gene (phosphoenolpyruvate carboxykinase and glucose-6 phosphatase) expression $^{52)}$. RBO also reduces the oxidative stress in pancreatic beta islet cells and increases insulin secretion from islet cells, subsequently increase the glucose utilization in liver and muscle cells. In this way, $\gamma$-oryzanol and tocotrienol can reduce total cholesterol, LDL-cholesterol, triglycerides, glucose level, and even bodyweight, subsequently triggering hyperlipidemia, hyperglycemia, hypertension, insulin resistance, and hyperinsulinemia ${ }^{53)}$. In vivo experiments showed that the bioactive component of RBO, $\gamma$-oryzanol at about 26-50 $(\mu \mathrm{mol} / \mathrm{L})$ could be effective in reducing cholesterol uptake and lipogenesis-related gene expression in human Caco-2 cells (HBT-37) and human hepatocellular carcinoma (HepG2) cells. $\gamma$-oryzanol consumption (300mg/day) has a positive effect on serum lipids, thyroid-stimulating hormone, and menopausal abnormalities $^{54,55)}$. We summarized in Fig. 2 , the proposed mechanism of how RBO reduces hyperglycemia and hyperlipidemia.

\section{Properties of RBO in Anticancer, Antioxidant, and Anti-inflammatory}

The bioactive components of RBO named $\gamma$-oryzanol and tocotrienol can inhibit cancer cells, specifically cycloartenyl ferulate and $\gamma$-tocotrienol could inhibit human colon carcinoma cell line (SW480) and subsequently accelerate programmed cell death in the initial stages of colorectal cancer. It has been believed that oryzanol and tocotrienols are safe and promising anticancer compounds because of their antioxidant capacity and anti-inflammatory properties and are more effective in cancer prevention than treatment $^{56,57)}$. Reactive oxygen species (ROS) and new reactive species or free radicals come from mitochondrial dysfunction and can cause metabolic abnormalities by oxidation of protein, lipids, and DNA, which is the leading cause of

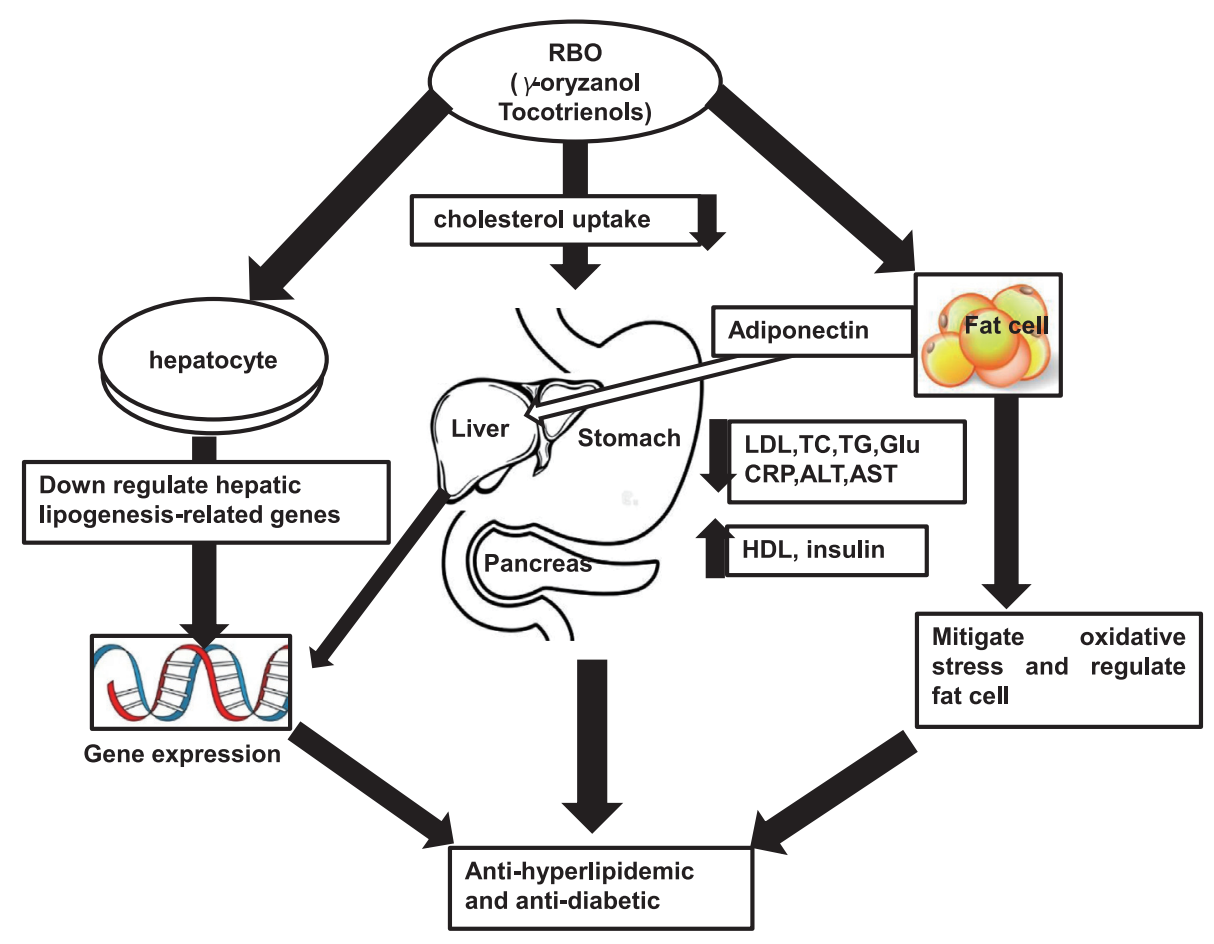

Fig. 2 Schematic summary of anti-lipidemic and anti-diabetic properties of RBO. 
chronic disease. The RBO can help to maintain metabolic homeostasis by modulating the related pathway in the responsive arena. One of the mechanisms is by inhibiting the pathway that activates the nuclear transcription factorkappa $\mathrm{B}(\mathrm{NF}-\kappa \mathrm{B})$, responsible for a variety of inflammatory diseases, and the other is by downregulating the tumor necrosis factor-alpha (TNF- $\alpha$ ) and can protect cell homeostasis $^{58)}$. Oryzanol and tocotrienol may improve mitochondrial functions and may prevent or slow down the progression and development of disease by protecting cellular DNA, lipid and protein oxidation. In some cases, tocotrienol exhibits stronger antioxidant activity than tocopherol, which is attributed to its high capacity to donate phenolic hydrogen to ROS and free radicals. Therefore, they can neutralize more ROS than $\gamma$-oryzanol and the $\gamma$-tocotrienol(-T3) component of RBO can promote tumor cell apoptosis via a chain of cascade reactions ${ }^{59-63)}$. The RBO has polyunsaturated essential fatty acids such as linoleic acid, which can exert an antitumor effect and ameliorate inflammation-induced colorectal cancer by activation of the PPAR- $\gamma$. Therefore, RBO bioactive compounds can exert anticancer, antioxidant, and anti-inflammatory effects through several pathways. We summarized in Fig. 3, the role of RBO in cancer and other chronic diseases that are caused by oxidation and inflammation. The $\gamma$-oryzanol and $\gamma$-tocotrienol can exert their action by trapping the oxidized molecule and neutralizing reactive oxygen species to inhibit ROS and new free radicals. On the other hand, they can activate the nuclear transcription factor $\mathrm{NF}-\mathrm{\kappa B}$ and inhibit protein kinase C, increase IL-10 and also inhibit TNF- $\alpha$ and inflam- matory cytokines and enzymes (IL-1, IL-6, IL-8, inducible nitric oxide synthase, and cyclooxygenase 2) by their antiinflammatory action. The most important role of RBO antitumor can be exerted through inducing death receptors activating caspase cascade reactions ${ }^{63-65)}$. Abnormal cell cycle protein expression can lead to uncontrolled cell proliferation, one of the causes of carcinoma. The molecules that target the cell cycle protein to reduce cell proliferation could be effective anticancer properties. The bioactive component, $\gamma$-tocotrienol, can inhibit human gastric adenocarcinoma SGC-7901 cell proliferation by arresting the cell cycle at the G0/G1 phase, whereas the $\gamma$-oryzanol inhibits both the G0/G1 phase and the resting G2/M phase. On the other hand, cancer is developed by the deregulation of cell apoptosis. The control of cancer cell development by promotion of cell apoptosis is one of the critical approaches to cancer treatment ${ }^{10)}$. The bioactive component of RBO (cycloartenyl ferulate, $\gamma$-tocotrienol) significantly induced cancer cell apoptosis via promoting the expression of proapoptotic proteins (Bax, Bcl-xl, and caspase-3, caspase-8, caspase-9). The mechanism of anticancer, antioxidant, and anti-inflammation of Bangladeshi RBO is still undetermined. Figure 3 summarized the proposed mechanism of Bangladeshi RBO for extensive research in vitro and in vivo for elucidation of anticancer properties.

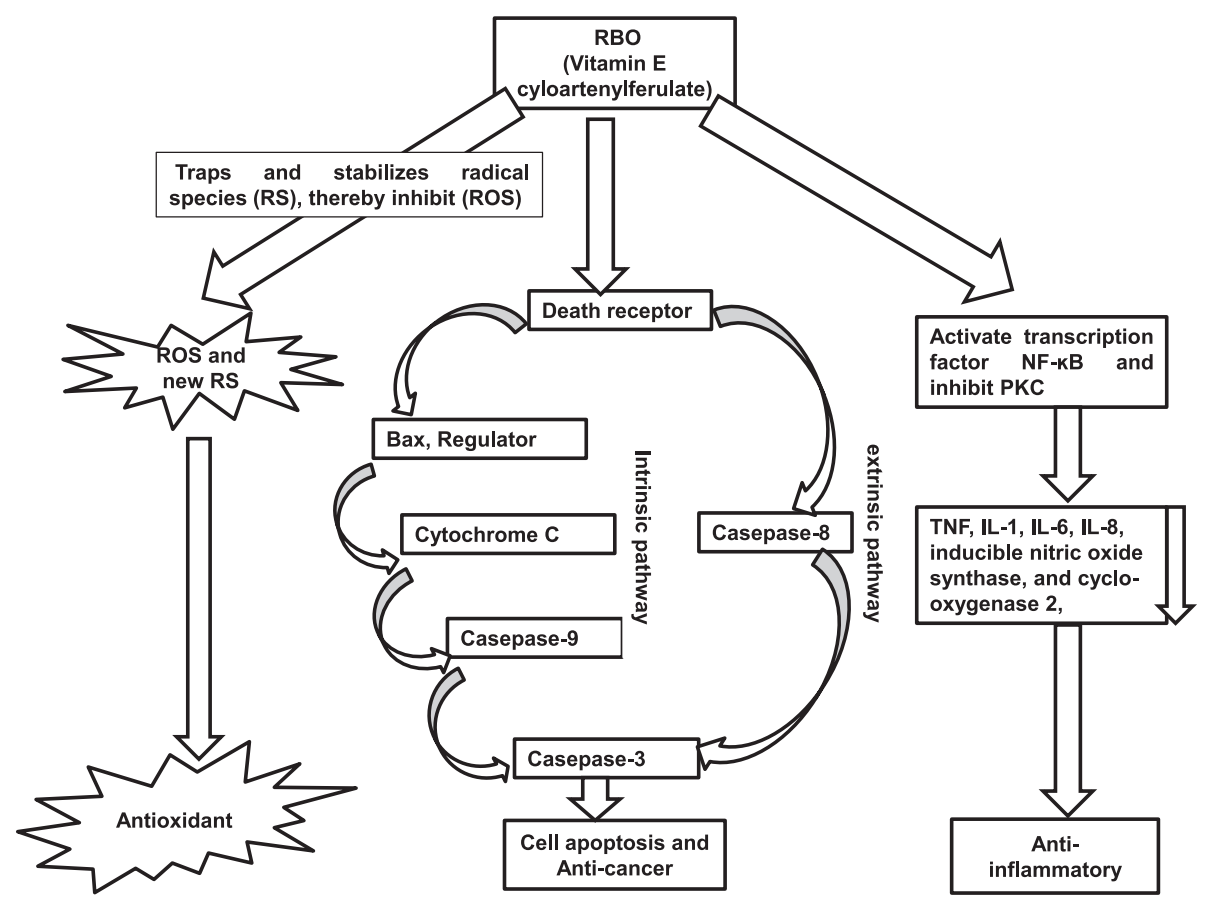

Fig. 3 Schematic summary of antioxidant, anti-inflammatory and anticancer properties of RBO. 


\section{Other Properties of RBO}

\subsection{Anti ulcerogenic function of RBO}

The prevalence of ulceration varies geographically and in food habits around the world. The high prevalence of ulceration was found in refined grains as a staple food consuming area, whereas a low prevalence was found in unrefined rice and wheat staple food consuming areas ${ }^{66)}$. RBO can reduce ulcer formation $66.75 \%$ by modulating gastric acid levels ${ }^{67)}$. Moreover, $\gamma$-oryzanol protects the gastric mucosa from ethanol-induced gastric lesions by increasing the level of gastric mucus ${ }^{67)}$. Different animal model experiments showed that RBO has ulcer protective activity due to its high level of phospholipid, sterol, and sterol ester fractions such as phosphatidylcholine(lethicin) and phosphatidylethanolamine (cephalin) predominantly. The sterol, $\beta$-sitosterol, stigmasterol, and an unidentified isomer of $\beta$-sitosterol showed protective activity against ulceration. The protective activity of RBO may also be of clinical importance in giving protection against the ulcerogenic effect $^{66-68)}$. Several studies have observed that hydroxycinnamic acid derivatives, such as curcumin and caffeic acid phenethyl ester, can inhibit NF- $\kappa$ B activity, one of the most important transcriptional factors in inflammation ${ }^{69,70)}$. A lot of related hydroxycinnamic acids, such as phytosterol ferulic acid esters, have been found in RBO. One animal experiment reported that phytosterol ferulates significantly inhibit inducible nitric oxide synthase(iNOS) expression and nitric oxide(NO) production in activated macrophages by interfering with $\mathrm{NF}-\kappa \mathrm{B}$ activation in dextran sulfate sodium (DSS)-induced colitis ${ }^{71)}$. Another study explained the inhibitory mechanism of phytosteryl ferulates on the NF-кB signaling pathway. RBO (Phytosteryl ferulates) can inhibit degradation of $\mathrm{I}-\mathrm{kB}$, resulting in the inhibition of $\mathrm{NF}-\kappa \mathrm{B}$ p65 nuclear translocation that is responsible for transcription of related genes ${ }^{72)}$. RBO can also reduce ROS through its scavenging and antioxidative abilities. Thus, the $\mathrm{RBO}$ could be a remedy for ulcerogenic treatment, but further experiments need to be explored.

\subsection{Neuroprotective function of RBO:}

$\mathrm{RBO}$ is the richest source of $\gamma$-oryzanol than any other vegetable oil. The RBO showed antioxidant and effective anti-proinflammatory effects on the cerebral milieu as indicated by a significant reduction in active glial cells and was found to improve synaptic and neuronal signaling. This is caused by $\gamma$-oryzanol, a compound that can cross the blood-brain barrier with its active form and affects brain function. Studies have shown that only $100 \mathrm{mg} / \mathrm{kg} /$ day, $\gamma$-oryzanol can modulate the hippocampal protein that is involved in synaptic and neuronal signaling, thereby functioning as a neuroprotector and antioxidant. On the other hand, $0.5 \% \gamma$-Oryzanol can control anxiety-like behavior by modulating the monoaminergic neuronal signaling pathway $^{73,74)}$. Thus, the bioactive component of RBO, $\gamma$-oryzanol, exhibits its candidature as a therapeutic moiety to improve cognitive behavior in neurodegenerative disorders. One study has observed that rice bran extracts significantly inhibited the release of prostaglandin E2 (PGE2) and free radical formation (8-iso-PGF2 $\alpha$ ) in LPS-activated primary microglia-a type of neuroglia (glial cell) located throughout the brain and spinal cord ${ }^{75)}$. Inhibition of PGE2 by rice bran extract $(\mathrm{RBE})$ was dependent on reduced cyclooxygenase-2(COX-2) and microsomal prostaglandin E synthase-1 (mPGES-1) immunoreactivity in microglia. It was also observed that treatment of activated microglia with RBE further enhanced the gene expression of the microglial M2 marker IL-10 and reduced the expression of pro-inflammatory M1 markers (TNF- $\alpha$, IL-1 $\beta$ ). Specific protective properties of $\mathrm{RBO}$ were observed in the postsynaptic receptor(D2) antagonist haloperidol-induced Tardive dyskinesia, which has potential implications in the treatment of schizophrenia and motor disorders ${ }^{76)}$. Nevertheless, the exact mechanism of the whole RBO's activity in cognitive development remains to be discovered.

\subsection{Immune system modulation by RBO:}

Immune system modulation, also called immunomodulation, is the process that involves the use of therapy to modify the immune response, often to prevent tissue damage resulting from an excessive response. The immune system is controlled both by direct interaction of several types of cells (lymphoid cells: B and T lymphocytes, T helper (Th) cells, natural killer (NK) cells; myeloid cells: neutrophils, basophils, monocytes, macrophages) and secreted molecules (immunoglobulins, cytokines: interleukins, colony-stimulating factors, growth factors, interferons, etc.) by them. RBO modulates the immune system by enhancing B-lymphocyte proliferation and TH1-type cytokines such as IL-2 or TNF- $\alpha$. Reduction of TH2 cytokine IL-4 and immunoglobulin E (IgE) levels suggested that antiallergenic properties of $\mathrm{RBO}^{77-79)}$, it has been observed that $\gamma$-oryzanol possesses sufficient potential for augmenting immune activity by cellular mediated mechanisms.

\section{Conclusion}

The RBO itself or therapeutically bioactive components both are safe and effective in ameliorating lifestyle-related diseases. This review suggested some possible beneficial effects of Bangladesh RBO such as anti-hyperglycemic and antilipidemic, antioxidative, anti-inflammatory, anti-cancer, anti-ulcerogenic, immune, and neurodegenerative disease prevention. RBO seems to be a very promising oil to reduce the growing incidence of non-communicable disease for 21st century's ailment. However, more research is needed to confirm the beneficial effects of RBO. Nevertheless, the exact mechanism of RBO in Bangladesh remains to be dis- 
covered.

\section{Acknowledgment}

We are particularly grateful to Professor Hitoshi Shirakawa for suggestions and constant inspiration for manuscript preparation. We are also grateful to Jashore University of Science and Technology for the logistic support of this review article.

\section{Reference}

1) Molina, J.; Sikora, M.; Garud, N.; Flowers, J.M.; Rubinstein, S.; Reynolds, A.; Purugganan, M.D. Molecular evidence for a single evolutionary origin of domesticated rice. Proc. Natl. Acad. Sci. USA 108, 8351-8356 (2011).

2) Pali, V. Rice bran oil-unique gift of nature: A review. Agri. Revi. 34, 288-294 (2013).

3) Garba, U.; Singanusong, R.; Jiamyangyuen, S.; Thongsook, T. Extraction and utilization of rice bran oil: A review. Rice Bran Oil Application: Pharma-Cosmetics, Nutraceuticals and Foods 24-25 August (2017).

4) Sharif, M.K.; Butt, M.S.; Anjum, F.M.; Khan, S.H. Rice bran: A novel functional ingredient. Crit. Rev. Food. Sci. Nutr. 54, 807-816 (2014).

5) Dunford, N.T. Chemistry of rice bran oil. in Rice. AOCS Press, pp. 1-18(2019).

6) Lai, O.M., Jacoby, J.J.; Leong, W.F.; Lai, W.T. Nutritional studies of rice bran oil. in Rice. AOCS Press, pp. 19-54 (2019).

7) Roger, E.J.; Rice, S.M.; Nicolosi, R.J.; Carpenter, D.R.; McClelland, C.A.; Romanczyk Jr, L.J. Identification and quantification of $\gamma$-oryzanol components and simultaneous assessment of tocols in rice bran oil. J. Am. Oil. Chem. Soc. 70, 301-307 (1993).

8) Sugihara, N.; Kanda, A.; Nakano, T.; Nakamura, T.; Igusa, $\mathrm{H}$; Hara, $\mathrm{S}$. Novel fractionation method for squalene and phytosterols contained in the deodorization distillate of rice bran oil. J. Oleo. Sci. 59, 65-70 (2010).

9) Lai, M.H.; Chen, Y.T.; Chen, Y.Y.; Chang, J.H.; Cheng, H.H.; Effects of rice bran oil on the blood lipids profiles and insulin resistance in type 2 diabetes patients. J. Clin. Biochem. Nutr. 51, 11-87(2011).

10) Yu, Y.; Zhang, J.; Wang, J.; Sun, B. The anti-cancer activity and potential clinical application of rice bran extracts and fermentation products. RSC Adv. 9, 1806018069 (2019).

11) de Deckere, E.A.; van, Amelsvoort, J.M.; McNeill, G.P.; Jones, P. Effects of conjugated linoleic acid (CLA) isomers on lipid levels and peroxisome proliferation in the hamster. Br. J. Nutr. 82, 309-317 (1999).

12) Berger, A.; Rein, D.; Schafer, A.; Monnad, I.; Gremaud, G; Lambelet, P.; Bertoli, C. Similar cholesterol-lowering properties of rice bran oil, with varied $\gamma$-oryzanol, in mildly hypercholesterolemic men. Eur. J. Nutr. 44, 163-173 (2005).

13) Zavoshy, R.; Noroozi, M.; Jahan Hashemi, H. Effect of lowcalorie diet with rice bran oil on cardiovascular risk factors in hyperlipidemic patients. J. Res. Med. Sci. 17, 626(2012).

14) Wang, T.; Khir, R.; Pan, Z.; Yuan, Q. Simultaneous rough rice drying and rice bran stabilization using infrared radiation heating. LWT-Food Sci. Technol. 78, 281-288(2017).

15) Jin, J.; Xie, D.; Chen, H.; Wang, X.; Jin, Q.; \& Wang, X. Production of rice bran oil with light color and high $\gamma$-Oryzanol content by multi-stage molecular distillation. J. Am. Oil. Chem. Soc. 93, 145-153(2016).

16) Alauddin, M.; Rahman, S.; Islam, J.; Shirakawa, H.; Komai, M.; Howlader, M.Z.H. Development of rice bran functional food and evaluation of its healthful properties. in Rice. AOCS Press, pp. 183-206 (2019).

17) Tong, C.; Bao, J. Rice lipids and rice bran oil. in Rice. AOCS Press, pp. 131-168(2019).

18) Lisa, S.A.; Khan, S.; Kabir, M.A.; Islam, F.; Mohajan, S.; Chowdhury, K. Quality and vitamin A status assessment of different commercial edible oil. Bangladesh $J$. Sci. Ind. Res. 54, 11-20 (2019).

19) Kahlon, T.S. Rice bran: Production, composition, functionality and food applications, physiological benefits, Chapter 14. in Fiber Ingredients Food Applications and Health Benefits (Cho, S.S.; Samuel, P. eds.). CRC Press, pp. 305-329 (2009).

20) Orsavova, J.; Misurcova, L.; Ambrozova, J.V.; Vicha, R.; Mlcek, J. Fatty acids composition of vegetable oils and its contribution to dietary energy intake and dependence of cardiovascular mortality on dietary intake of fatty acids. Int. J. Mol. Sci. 16, 12871-12890 (2015).

21） Almeida, D.T.D.; Viana, T.V.; Costa, M.M.; Silva, C.D.S.; Feitosa, S. Effects of different storage conditions on the oxidative stability of crude and refined palm oil, olein and stearin(Elaeis guineensis). J. Food Sci. Technol. 39 (Suppl.1), 211-217(2019).

22) Sahasrabudhe, S.N.; Veronica, R.; Meghan, M.; Farkas, B.E. Density, viscosity, and surface tension of five vegetable oils at elevated temperatures: Measurement and modeling. Int. J. Food. Prop. 20, 1965-1981 (2017).

23) Chebet, J.; Kinyanjui, T.; Cheplogoi, P.K. Impact of frying on iodine value of vegetable oils before and after deep frying in different types of food in Kenya. J. Sci. Ind. Res. 5, 193-196 (2016).

24) Shahidi, F.; de Camargo, A.C. Tocopherols and tocotrienols in common and emerging dietary dources: Occurrence, applications and health benefits. Int. J. Mol. 
Sci. 17, 1745(2016)

25) Ahsan, H.; Ahad, A.; Siddiqui, W.A. A review of characterization of tocotrienols from plant oils and foods. $J$. Chem. Biol. 20, 45-59 (2015).

26) Nourbehesht, N.; Shekarchizadeh, H.; Soltanizadeh, N. Investigation of stability, consistency, and oil oxidation of emulsion filled gel prepared by inulin and rice bran oil using ultrasonic radiation. Ultrason. Sonochem. 42, 585-593 (2018).

27) Sohail, M.; Rakha, A.; Butt, M. S.; Iqbal, M. J.; Rashid, S. Rice bran nutraceutics: A comprehensive review. Crit. Rev. Food. Sci. Nutr. 57, 3771-3780(2017).

28) Mishra, R.; Sharma, H.K.; Sarkar, B.C.; Singh, C. Thermal oxidation of rice bran oil during oven test and microwave heating. J. Food Sci. Technol. 49, 221-227 (2012).

29) Gunstone, F.D; Harwood, J.L. The Lipids Handbook (3rd ed.). CRC Press, p. 68(2007).

30) Shakib, M.C.; Gabrial, S.; Gabrial, G. Rice bran oil compared to atorvastatin for treatment of dyslipidemia in patients with type 2 diabetes. Maced. J. Medical Sci. 7, 95-102 (2014).

31) K. Chowdhury, L. A. Banu, S. Khan and A. Latif Studies on the fatty acid composition of edible oil. Bangladesh J. Sci. Ind. Res. 42, 311-316(2007).

32) Konuskan, D.B.; Arslan, M.; Oksuz, A. Physicochemical properties of cold pressed sunflower, peanut, rapeseed, mustard, and olive oils grown in the Eastern Mediterranean region. Saudi. J. Biol. Sci. 26, 340$344(2019)$.

33) Azeman, N.H.; Yusof, N.A.; Othman, A.I. Detection of free fatty acid in crude palm oil. Asian Journal of Chemistry 27, 1569 (2015).

34) Finglas, P.M.; Roe, M.A.; Pinchen, H.M.; Berry, R.; Church, S.M. et al. McCance and Widdowson's The Composition of Foods Seventh Summary edition. Royal Society of Chemistry, Cambridge (2015). https:// pubs.rsc.org/is/content/chapter/9781849737562fp001/978-1-84973-636-7/unauth

35) Manna, S.; Sharma, H.B.; Vyas, S.; Kumar, J. Comparison of mustard oil and ghee consumption on the history of coronary heart disease in urban population of India. J. Clin. Diagn. Res. 10 (10), OC01-OC05 (2016).

36) Yang, R.; Xue, L.; Zhang, L.; Wang, X.; Qi, X.; Jiang, J.; Li, P. Phytosterol contents of edible oils and their contributions to estimated phytosterol intake in the Chinese diet. Foods 8 (8), 334 (2019).

37) Mortuza, M.G. Tocopherol and sterol content of some rapeseed/mustard cultivars developed in Bangladesh. Pakistan Journal of Biological Sciences 9, 18121816 (2006).

38) Tsouko, E.; Alexandri, M.; Vieira Fernandes, K.; Guimarães Freire, D.M.; Mallouchos, A.; Koutinas, A.A. Extraction of phenolic compounds from palm oil pro- cessing residues and their application as antioxidants. Food Technol. Biotechnol. 57, 29-38(2019).

39) Prata, R.; Pereira, J.A.; Rodrigues, N.; Dias, L.G.; Veloso, A.C.; Casal, S.; Peres, A.M. Olive oil total phenolic contents and sensory sensations trends during oven and microwave heating processes and their discrimination using an electronic tongue. J. Food Qual. 2018, 7826428(2018).

40) Rahman, M.A.; Ferdous, J.; Hosen, S.; Hasan, M.; Parvin, A.; Shozib, H.B. Hypercholesterolemia ameliorating effect of Bangladeshi high yield variety rice bran oil. Int. J. Adv. Biol. Biomed. Res. 9, 48-57 (2021).

41) Ali, S.L.; Ubaidullah, M.R.; Hasan, M.M.; Hossain, M.L. Prospect of rice bran oil industries in Bangladesh. Bangladesh Journal of Tariff and Trade 3, 14-33 (2015).

42) Quaiyum, A.; Nahar, S.G.; Ubaidullah, M.R.; Hasan, M.M.; Latif, M.A. An analysis of assistance to edible oil manufacturing industry and examining export possibilities. Bangladesh Journal of Tariff and Trade 1 (2), 6-28 (2015).

43) Shozib, H.B. Post-harvest loss minimization of rice bran for quality bran oil production in Bangladesh. EC Nutrition 13, 667-675 (2018).

44) Endo, Y.; Nakagawa, K. Differences in the compositions of vitamin E tocochromanol(tocopherol and tocotrienol) in rice bran oils produced in Japan and other countries. J. Oleo. Sci. 70, 503-507(2021).

45) Rahman, M.; Nurul, A.; Mondal, Md.; Hossan, M.; Seatara, K.; Khan, G.R.M.; Md.I. Lipid, fatty acid and glyceride composition of five different varieties of rice bran oil. Bangladesh Research Publications Journal 9(2), 111-115(2013).

46) Rahman, M.A.; Ferdous, J.; Hosen, S.; Hasan, M.; Parvin, A.; Shozib, H.B. Hypercholesterolemia ameliorating effect of Bangladeshi high yield variety rice bran oil. Int. J. Adv. Biol. Biomed. Res. 9, 48-57 (2021).

47) Islam, N.; Islam, M.S.; Alam, M.J.; Islam, M.K.; Ahmad, N. Effects of soybean and rice bran oil on hemato-biochemical parameters in mice. Res. Agric. Livest. Fish. 5, 3(2018)

48) Kozuka, C.; Yabiku, K.; Sunagawa, S.; Ueda, R.; Taira, S.I.; Ohshiro, H.; Masuzaki, H. Brown rice and its component, $\gamma$-oryzanol, attenuate the preference for highfat diet by decreasing hypothalamic endoplasmic reticulum stress in mice. Diabetes 61, 3084-3093(2012).

49) Yamauchi, T.; Kamon, J.; Minokoshi, Y.A.; Ito, Y.; Waki, H.; Uchida, S.; Kadowaki, T. Adiponectin stimulates glucose utilization and fatty-acid oxidation by activating AMP-activated protein kinase. Nature Med. 8, 1288-1295 (2002).

50) Barthel, A.; Schmoll, D. Novel concepts in insulin regulation of hepatic gluconeogenesis. Am. J. Physiol. Endocrinol. Metab. 285, E685-692 (2003). 
51) Ohara, K.; Kiyotani, Y.; Uchida, A.; Nagasaka, R.; Maehara, H.; Kanemoto, S.; Ushio, H. Oral administration of $\gamma$-aminobutyric acid and $\gamma$-Oryzanol prevents stressinduced hypoadiponectinemia. Phytomedicine 18, 655-660 (2011).

52) Combs, T.P.; Berg, A.H.; Obici, S.; Scherer, P.E.; Rossetti, L. Endogenous glucose production is inhibited by the adipose-derived protein Acrp30. J. Clin. Invest. 108, 1875-1881 (2001).

53) Minatel, I.O.; Francisqueti, F.V.; Corrêa, C.R.; Lima, G.P.P. Antioxidant activity of $\gamma$-oryzanol: A complex network of interactions. Int. J. Mol. Sci. 17, 1107 (2016).

54) Mäkynen, K.; Chitchumroonchokchai, C.; Adisakwattana, S.; Failla, M.; Ariyapitipun, T. Effect of gamma- $\gamma$-oryzanol on the bioaccessibility and synthesis of cholesterol. Eur. Rev. Med. Pharmacol. Sci. 16, 49-56 (2012).

55) Cicero, A.F.G.; Gaddi, A. Rice bran oil and $\gamma$-oryzanol in the treatment of hyperlipoproteinemias and other conditions. Phytother. Res. 15, 277-289 (2001).

56) Kong, C.K.; Lam, W.S.; Chiu, L.C.; Ooi, V.E.; Sun, S.S.; Wong, Y.S. A rice bran polyphenol, cycloartenyl ferulate, elicits apoptosis in human colorectal adenocarcinoma SW480 and sensitizes metastatic SW620 cells to TRAIL-induced apoptosis. Biochem. Pharmacol. 77, 1487-1496 (2009).

57) Szcześniak, K.A.; Ostaszewski, P.; Ciecierska, A.; Sadkowski, T. Investigation of nutriactive phytochemical $\gamma$-oryzanol in experimental animal models. J. Anim. Physiol. Anim. Nutr. (Berl.) 100,601 (2016).

58) Suganya, K.S.; Govindaraju, K.; Kumar, V.G.; Karthick, V.; Parthasarathy, K. Pectin mediated gold nanoparticles induces apoptosis in mammary adenocarcinoma cell lines. Int J. Biol. Macromol. 93, 1030-1040 (2016).

59) Ellis, R.; Seal, M.L.; Adamson, C.; Beare, R.; Simmons, J.G. et al. Brain connectivity networks and longitudinal trajectories of depression symptoms in adolescence. Psychiatry Res. 21, 62-69(2016).

60) Serafini, M.; Peluso, I. Functional foods for health: the interrelated antioxidant and anti-inflammatory role of fruits, vegetables, herbs, spices and cocoa in humans. Curr. Pharm. Des. 22, 6701-6715(2016).

61) Hagl, S.; Berressem, D.; Grewal, R.; Sus, N.; Frank, J.; Eckert, G.P. Rice bran extract improves mitochondrial dysfunction in brains of aged NMRI mice. Nutr. Neurosci. 19, 1-10 (2016).

62) Kannappan, R.; Yadav, V.R; Aggarwal, B.B. $\gamma$-Tocotrienols but not $\gamma$-tocopherol blocks STAT3 cell signaling pathway through induction of protein-tyrosine phosphatase SHP-1 and sensitizes tumor cells to chemotherapeutic agents. J. Biol. Chem. 285, 3352033528(2010).
63) Kannappan, R.; Ravindran, J.; Prasad, S. Gamma-tocotrienols promotes TRAIL-induced apoptosis through reactive oxygen species/extracellular signal-regulated kinase/p53-mediated upregulation of death receptors. Mol. Cancer. Ther. 9, 2196-2207 (2010).

64) Liang, Y.; Gao, Y.; Lin, Q.; Luo, F.; Wu, W.; Lu, Q.; Liu, Y. A review of research progress on the bioactive ingredients and physiological activities of rice bran oil. Eur. Food. Res. Technol. 238, 169-176 (2014).

65) Lee, S.; Yu, S.; Park, H.J.; Jung, J.; Go, G.W.; Kim, W. Rice bran oil ameliorates inflammatory responses by enhancing mitochondrial respiration in murine macrophages. PlosOne 14, e0222857 (2019).

66) Tovey, F.I. Role of dietary phospholipids and phytosterols in protection against peptic ulceration as shown by experiments on rats. World J. Gastroenterol. 21, $1377(2015)$.

67) Trinovita, E.; Chany Saputri, F.; Mun'im, A. Potential gastroprotective activity of rice bran (Oryza sativa L.) extracted by ionic liquid-microwave-assisted extraction against ethanol-induced acute gastric ulcers in rat model. Sci. Pharm. 86 (3), 35 (2018).

68) Forner, L.G.; Hernández, B.B.; Bermúdez, M.A.C.; Matíez, J.A..; Pérez, E.F.P. et al. Antiulcerogenic properties of bran rice oil in rats. Rev. Esp. Enferm. Apar. Dig. 75, 225-230 (1989).

69) Singh, S.; Aggarwal, B.B. Activation of transcription factor NF-kappa B is supressed by curcumin (diferuloylmethane). J. Biol. Chem. 270, 24995-5000 (1995).

70) Natarajan, K.; Singh, S.; Burke, T.R.; Jr. Grunberger, D.; Aggarwal, B.B.; Caffeic acid phenethyl ester is a potent and specific inhibitor of activation of nuclear transcription B. Proc. Natl. Acad. Sci. USA 93, 90909005 (1996).

71) Nagasaka, R.; Chotimarkorn, C.; Shafiqul, I.M.; Hori, M.; Ozaki, H.; Ushio, H. Anti-inflammatory effects of hydroxycinnamic acid derivatives. Biochem. Biophys. Res. Commun. 358, 615-619(2007).

72) Islam, M.S.; Yoshida, H.; Matsuki, N.; Ono, K.; Nagasaka, R.; Ushio, H.; Guo, Y.; Hiramatsu, T.; Hosoya, T.; Murata, T.; Hori, M.; Ozaki, H. Antioxidant, free radical-scavenging, and NF- $\kappa \mathrm{B}$-inhibitory activities of phytosteryl ferulates: structure-activity studies. J. Pharmacol. Sci. 111, 328-337(2009).

73) Rungratanawanich, W.; Cenini, G.; Mastinu, A.; Sylvester, M.; Wilkening, A.; Abate, G.; Uberti, D. $\gamma$-Oryzano improves cognitive function and modulates hippocampal proteome in mice. Nutrients 11, 753(2019).

74) Akter, S.; Uddin, K.R.; Sasaki, H.; Lyu, Y.; Shibata, S. $\gamma$-Oryzanol impairs alcohol-induced anxiety-like behavior in mice via upregulation of central monoamines associated with Bdnf and Il-1 $\beta$ signaling. Sci. Rep. 10, $1-13(2020)$.

75) Bhatia, H.S.; Baron, J.; Hagl, S. Rice bran derivatives 
alleviate microglia activation: Possible involvement of MAPK pathway. J. Neuroinflamm. 13, 148 (2016).

76) Samad, N.; Haleem, D.J. Antioxidant effects of rice bran oil mitigate repeated haloperidol-induced tardive dyskinesia in male rats. Metab. Brain Dis. 32, 10991107(2017).

77) Grigore, A. Plant phenolic compounds as immunomodulatory agents. Phenolic compounds-Biological activity. London, UK. Intech Open 75-98 (2017).

78) Sierra, S.; Lara-Villoslada, F.; Olivares, M. Increased immune response in mice consuming rice bran oil. Eur. J. Nutr. 44, 509-516 (2005).
79) Ghatak, S.B.; Panchal, S.J. Investigation of the immunomodulatory potential of $\gamma$-oryzanol isolated from crude rice bran oil in experimental animal models. Phytother. Res. 26, 1701-1708(2012).

CC BY 4.0 (Attribution 4.0 International). This license allows users to share and adapt an article, even commercially, as long as appropriate credit is given. That is, this license lets others copy, distribute, remix, and build upon the Article, even commercially, provided the original source and Authors are credited. 